\title{
Water diffusion in rough carbon nanotubes
}

Cite as: J. Chem. Phys. 152, 024708 (2020); https://doi.org/10.1063/1.5129394

Submitted: 27 September 2019 . Accepted: 27 December 2019 . Published Online: 13 January 2020

Bruno H. S. Mendonça, (D) Patricia Ternes, Evy Salcedo, (iD) Alan B. de Oliveira, and (D) Marcia C. Barbosa
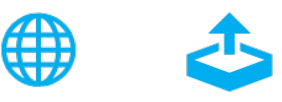

Export Citation



View Online

\section{ARTICLES YOU MAY BE INTERESTED IN}

On the transferability of ion parameters to the TIP4P/2005 water model using molecular dynamics simulations

The Journal of Chemical Physics 152, 024501 (2020); https://doi.org/10.1063/1.5124448

How methane hydrate recovers at very high pressure the hexagonal ice structure

The Journal of Chemical Physics 152, 024504 (2020); https://doi.org/10.1063/1.5129617

An analysis of radical diffusion in ionic liquids in terms of free volume theory

The Journal of Chemical Physics 152, 024502 (2020); https://doi.org/10.1063/1.5138130

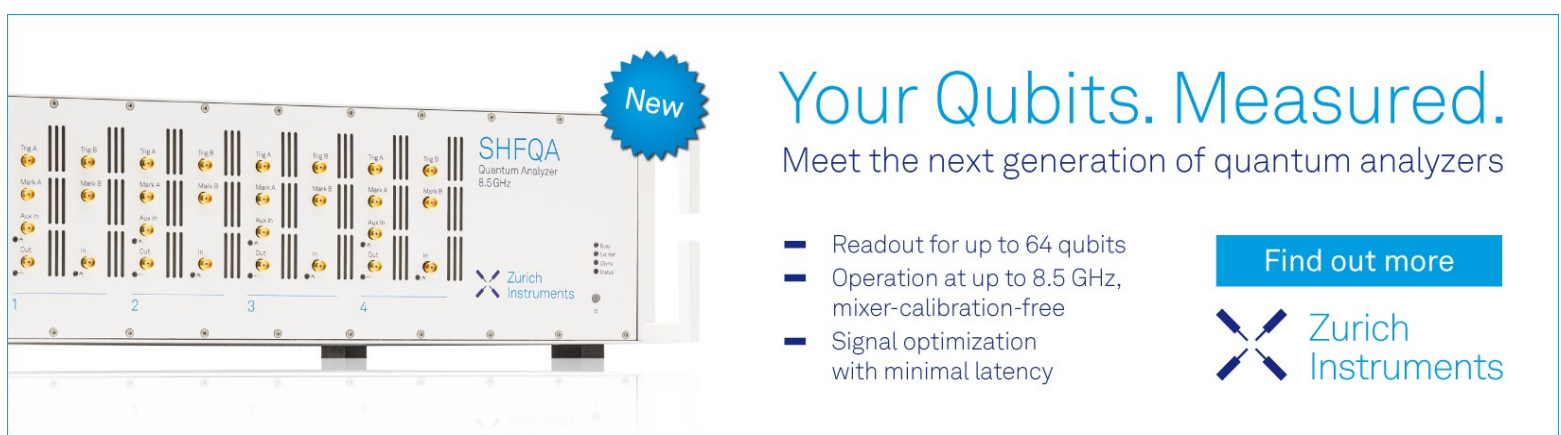




\title{
Water diffusion in rough carbon nanotubes
}

\author{
Cite as: J. Chem. Phys. 152, 024708 (2020); doi: 10.1063/1.5129394 \\ Submitted: 27 September 2019 - Accepted: 27 December 2019 • \\ Published Online: 13 January 2020
}

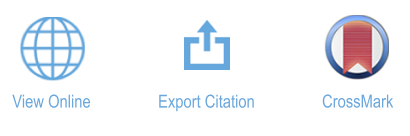

\section{Bruno H. S. Mendonça, ${ }^{1, a)}$ (iD Patricia Ternes, ${ }^{2}$ (D) Evy Salcedo, ${ }^{3}$ Alan B. de Oliveira, ${ }^{4}$ (iD) and Marcia C. Barbosa}

\author{
AFFILIATIONS \\ ${ }^{1}$ Instituto de Física, Universidade Federal do Rio Grande do Sul, Porto Alegre, RS 91501-970, Brazil \\ ${ }^{2}$ Campus São Bento do Sul, Instituto Federal Catarinense, São Bento do Sul, SC 89283-064, Brazil \\ ${ }^{3}$ Coordenadoria Especial de Física, Química e Matemática, Universidade Federal de Santa Catarina, Araranguá, \\ SC 88905-120, Brazil \\ ${ }^{4}$ Departamento de Física, Universidade Federal de Ouro Preto, Ouro Preto, MG, 35400-000, Brazil
}

a) Electronic mail: brunnohennrique13@gmail.com

\begin{abstract}
We use molecular dynamics simulations to study the diffusion of water inside deformed carbon nanotubes with different degrees of deformation at $300 \mathrm{~K}$. We found that the number of hydrogen bonds that water forms depends on nanotube topology, leading to enhancement or suppression of water diffusion. The simulation results reveal that more realistic nanotubes should be considered to understand the confined water diffusion behavior, at least for the narrowest nanotubes, when the interaction between water molecules and carbon atoms is relevant.
\end{abstract}

Published under license by AIP Publishing. https://doi.org/10.1063/1.5129394

\section{INTRODUCTION}

Physical and chemical properties of materials change dramatically depending on the confining size and geometry. ${ }^{1,2}$ For large systems, in the thermodynamic limit, surface effects are irrelevant when computing bulk properties. This is not the case of nanoscale confining geometries where volumetric and surface interactions have a similar order of magnitude. Then, the nature of the surface becomes a relevant factor for computing thermodynamic and dynamic properties of the confined fluid. One example of a surface impact on the fluid phase is water confined in carbon nanotubes (CNTs). Studies focusing fluid transport in carbon nanotubes are ubiquitous. ${ }^{3-5}$ Even though water transport properties in perfect nanotubes already present a number of interesting phenomena, deformations in nanopores change the degree of confinement that impacts the transport properties. These changes are present both in artificial and biological systems. For instance, deformations are relevant for the study of DNA sequencing, ${ }^{6}$ carbon nanotubes as nanosyringes, ${ }^{7}$ and nanothermometers.

Water exhibits a number of anomalous properties already in the bulk. When confined in nanostructures, additional anomalies arise. ${ }^{9-15}$ Water confined in carbon nanotubes exhibits a nonmonotonic behavior diffusion coefficient. For diameters above $6 \mathrm{~nm}$, the diffusion coefficient approaches the bulk water value. ${ }^{12}$ For diameters smaller than $6 \mathrm{~nm}$, the diffusion coefficient increases slightly as the CNT diameter decreases, reaching a maximum value for CNT diameters close to $2.5 \mathrm{~nm} .^{12}$ For even smaller diameters, the diffusion coefficient decreases with decreasing diameter, reaching a minimum value for diameters close to $1.2 \mathrm{~nm}$, followed by a significant increase in diffusion in the narrowest nanotubes. This nonmonotonic behavior was observed in simulations, ${ }^{16-18}$ and this high mobility was observed in experiments. ${ }^{19}$

The diffusion coefficient of nanoconfined water depends on two competing interactions: hydrogen bonds (HBs) between water molecules and the water-wall interaction. While the water hydrogen bonds decrease the energy by forming a tetrahedral network, which decreases the mobility, the water-wall interactions are less attractive molecules, creating dangling bonds, which privilege their mobility. Therefore, the precise nature of the water-wall interaction can affect the diffusion. For instance, theoretical and experimental works have also shown that different tube chiralities affect the confined water distribution inside carbon nanotubes and, consequently, its diffusion. ${ }^{20-22}$

Some studies indicate that armchair nanotubes have larger diffusivity when compared with the zigzag. ${ }^{11,23}$ These studies, however, were made of very small tubes and the diffusion was computed for short times, which might have affected their results. The changes in the length of the tubes produce a large impact on the mobility. ${ }^{9,1}$ 
This suggests that comparison between different chiralities should be performed for larger tubes.

Since the water diffusion inside carbon nanotubes depends strongly on the nanotube diameter, ${ }^{24,25}$ the defects which decrease the nanotube radius might impact the mobility of the water. In the production of the nanotubes, these defects and contaminations are expected. ${ }^{26}$ The resulting distortions from the perfect nanotube structure might lead to changes in the thermodynamic and dynamic behavior of the confined fluid.

Recently, a theoretical study analyzed the impact of a uniformed deformation in the diffusion coefficient of confined water. This study employed the TIP4P/2005 water model confined in a kneaded armchair nanotube. ${ }^{27}$ This specific type of deformation leads to the reduction in the lateral space which enhanced the number of hydrogen bonds and consequently reduced the water diffusion coefficient for almost all $(n, n)$ nanotubes. The exception was $(9,9)$ CNT where water has a near zero diffusion when the nanotube has zero deformation. In this particular case, deformation promotes the disruption of the hydrogen bond network. The icelike structures formed for the $(9,9)$ perfect nanotube melts by the compression, improving water mobility. Even though interesting, the study of water inside kneaded nanotubes is not realistic since it preserves the axial symmetry while nanotubes with randomly distributed deformations, wrinkled nanotubes, do not exhibit any specific symmetry. ${ }^{28,29}$

In this paper, we analyze through molecular dynamics (MD) simulations the effects of nonuniform deformations and chirality in the mobility of confined water inside carbon nanotubes. We compare the diffusion coefficient for the TIP4P/2005 water model when the liquid is confined in an armchair and zigzag wrinkled nanotubes. Our results for the two types of nanotubes are analyzed for perfect, kneaded, and wrinkled tubes. The remaining of this paper goes as follows. In Sec. II, the model is presented. Results are discussed in Sec. III, while conclusions are shown in Sec. IV.

\section{MODEL AND METHODS}

We studied the structural and dynamic behavior of water confined in carbon nanotubes with two different types of deformation: kneaded and wrinkled. In order to produce the kneaded nanotubes, a perfect nanotube was uniformly kneaded in the $y$ direction until the nanotube reached an elliptical cylinder shape with an eccentricity of 0.8 , as illustrated in Fig. 1. ${ }^{27}$ The deformation imposed to the kneaded nanotube is characterized by the eccentricity,

$$
e=\sqrt{1-\frac{b^{2}}{a^{2}}}
$$

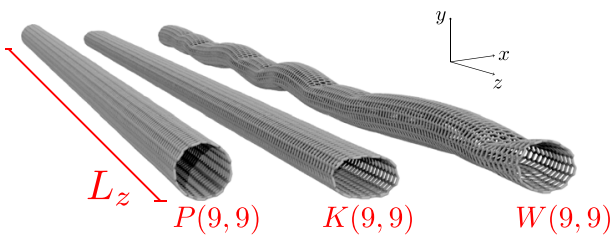

FIG. 1. Snapshot of perfect, $P(9,9)$; kneaded, $K(9,9)$; and wrinkled, $W(9,9)$ nanotubes. where $b$ is the smaller semiaxis and $a$ is the largest semiaxis. The second process was to disorderly compress the nanotube in the $y$ direction until it forms nonuniform wrinkles. Wrinkles in the systems were created in a disorderly manner, but as the number of wrinkles was small, the size distribution of the segments between two wrinkles was the same for nanotubes of the same diameter. In average, each nanotube was compressed in 5 different $z$ positions, with values of $e$ ranging from 0.0 to 0.8 .

In addition, for comparison purposes, perfectly structured nanotubes were also produced. The perfectly symmetrical nanotubes are characterized by an eccentricity of 0.0 . Figure 1 represents the three types of nanotubes used: perfect, kneaded, and wrinkled.

In order to illustrate the difference in water behavior due to structural changes in the $(n, m)$ nanotube, two different diameters of armchair $(n=m)$ and zigzag $(m=0)$ carbon nanotubes were used. Details of the structure of each nanotube are given in Table I. Note that $(9,9)$ and $(16,0)$ CNTs have approximately the same diameter. This is also the case for the $(12,12)$ and $(21,0)$ nanotubes.

Molecular dynamics (MD) simulations at a constant number of particles $N$, volume $V$, and temperature $T$ were employed to investigate the diffusion of the TIP4P/2005 water model. ${ }^{30}$ Periodic boundary conditions in the tube axial $(z)$ direction and a cutoff radius of $12 \AA$ were used. We represented the nonbonded interactions (carbon-oxygen) by the Lennard-Jones potential with parameters $\varepsilon_{\mathrm{CO}}=0.11831 \mathrm{kcal} / \mathrm{mol}$ and $\sigma_{\mathrm{CO}}=3.28 \AA^{3}$. The interaction between carbon and hydrogen was set to zero.

The water density inside carbon nanotubes was determined considering the excluded volume due to the Lennard-Jones interaction between carbon in the nanotubes and oxygen atoms in the water. Therefore, the effective density will be $\rho=4 M /$ $\left[\pi\left(d-\sigma_{\mathrm{CO}}\right)^{2} L_{z}\right]$, where $M$ is the total water mass into the tubes, while $L_{z}$ is the nanotube length. ${ }^{31}$

Simulations were carried out with the LAMMPS package. ${ }^{32} \mathrm{We}$ employed the particle-particle particle-mesh method to compute the long-range Coulomb interactions. This method treats long range interactions and the Colombian field of real charges in a way that can interfere with your own images. ${ }^{33}$ We work around this issue by creating an $\mathrm{x}-\mathrm{y}$ simulation box around $100 \mathrm{~nm}$ for all nanotubes, preventing the carbon nanotube from interacting with its own images and avoiding overlapping of virtual images with real images, minimizing possible errors in method application. The structure of water molecules was constrained through the SHAKE algorithm with a tolerance of $1 \times 10^{-4}$. The water temperature was maintained at $300 \mathrm{~K}$ through the Nosé-Hoover thermostat with a damping time of $100 \mathrm{fs}$ and $1 \mathrm{fs}$ time interval. In all simulations, we keep the nanotubes rigid and keep them from getting off the plane. This procedure has

TABLE I. Parameters for perfect carbon nanotubes.

\begin{tabular}{lcccc}
\hline \hline $\mathrm{CNT}$ & $\mathrm{d}(\mathrm{nm})$ & $\mathrm{L}_{z}(\mathrm{~nm})$ & $\mathrm{H}_{2} \mathrm{O}$ & $\rho\left(\mathrm{g} / \mathrm{cm}^{3}\right)$ \\
\hline$(9,9)$ & 1.22 & 50.5 & 908 & 0.92 \\
$(12,12)$ & 1.63 & 22.5 & 901 & 0.94 \\
$(16,0)$ & 1.25 & 50.5 & 908 & 0.80 \\
$(21,0)$ & 1.64 & 22.9 & 901 & 0.86 \\
\hline \hline
\end{tabular}


been employed in several similar simulations which have shown that considering the nanotube as a rigid system is a very reasonable approximation when compared to the case where the thermostat is applied throughout the system. ${ }^{12,34,35}$ The system was equilibrated during $5 \mathrm{~ns}$, and then, properties were stored every $0.01 \mathrm{~ns}$ during 5 $\mathrm{ns}$, giving a total simulation time of $10 \mathrm{~ns}$.

Due to system dimensions, the diffusion is minimal in the radial direction and only the axial diffusion is considered. It is determined through the one dimensional Einstein relation as

$$
D_{z}=\lim _{\tau \rightarrow \infty} \frac{1}{2} \frac{d}{d \tau}\left\langle z^{2}(\tau)\right\rangle,
$$

where $\left\langle z^{2}(\tau)\right\rangle=\left\langle\left[z\left(\tau_{0}-\tau\right)-z\left(\tau_{0}\right)\right]^{2}\right\rangle$ is the mean square displacement (MSD) in the axial direction.

In order to characterize the structure of water, we calculated the number of hydrogen bonds (HBs) and also made colormaps for oxygen occurrence in the $x y, x z$, and $y z$ planes. Hydrogen bonds were computed if both of the following geometrical criteria were satisfied:

$$
\begin{gathered}
\alpha \leq 30^{\circ}, \\
\left|\vec{r}_{\mathrm{OO}}\right| \leq 3.50 \AA,
\end{gathered}
$$

where $\alpha$ is the $\mathrm{OH} \cdots \mathrm{O}$ angle and $\left|\vec{r}_{\mathrm{OO}}\right|$ is the distance between two oxygen atoms.

Oxygen occurrence colormaps were obtained by dividing the correspondent plane in square bins of $0.1 \AA$ length and counting the number of oxygen atoms in each square. Higher oxygen densities are represented in red, while low densities tend to have darker blue tones.

\section{RESULTS AND DISCUSSION}

First, we compare the mobility of water inside perfect armchair and zigzag nanotubes for different diameters. Figure 2 shows that the water mobility is not strongly affected by the chirality of the nanotube for different diameters with the exception of the $(9,9)$ and $(16,0)$ nanotubes. This diameter is quite distinct when compared with smaller and larger nanotubes because just one water layer close to the wall is formed. This water layer behaves quite different depending on the chirality. While the water diffusion in the armchair

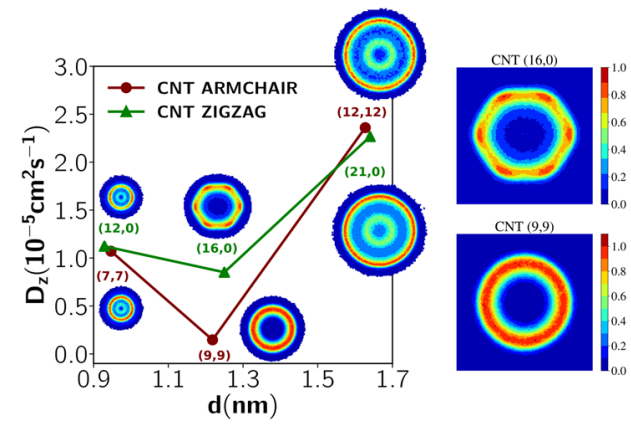

FIG. 2. Left panel: Diffusion coefficient vs nanotube diameter for perfect $P(n, m)$ armchair and zigzag nanotubes. Right panel: $x y$ colormaps show the density of water inside the nanotubes.
$(9,9)$ nanotube is almost null, nonzero diffusion is observed in the zigzag case. The density colormap illustrated in Fig. 2 shows that for the $(9,9)$ nanotube, water molecules are uniformly distributed in the vicinity of the wall. For the $(16,0)$ nanotube, the fluid molecules assume a hexagonal distribution at the wall boundary. The difference in the water distribution in the two types of nanotubes is due to the differences in the wall structures, as shown in Fig. 3, combined with the hydrophobic nature of the carbon-water interaction. The water molecules form hydrogen bonds but avoid being close to the carbon molecules.

For the zigzag nanotube, one hydrogen bond can be formed between two water molecules located at the middle of two neighbor carbon rings at the same $z$ coordinate, forming a ring. For the armchair, the hydrogen bond links water in the middle of two neighbor carbon rings but located at different $z$ coordinates, forming a spiral. The armchair spiral-like structure produces a more connected hydrogen bond network when compared with the ringlike structure of the zigzag nanotubes, resulting in a lower mobility of the armchair $(9,9)$ nanotube.

Next, we address the question of how nanotube deformations affect the water diffusion coefficient. In particular, we analyze the difference in water diffusion for armchair and zigzag tubes when they change from perfect to wrinkled and to kneaded. We analyze two nanotube diameters for each chirality: $(9,9)$ and $(12,12)$ for the armchair and $(16,0)$ and $(21,0)$ for the zigzag nanotubes. The diameter selection was made to test how the compression affects the number of layers and the distinct mobility between the two chiralities.

Deformation changes the way water molecules connect and move within the carbon nanotube, facilitating or hindering the formation of hydrogen bonds. This effect directly affects the diffusion of confined water molecules in the nanotube. If we analyze this effect locally, we can observe that curvature decreases the number of hydrogen bonds, which can be seen in Fig. 4. Figure 4 shows that for the $(12,12)$ armchair nanotube case, deformation decreases axial diffusion by increasing the number of hydrogen bonds as confirmed by previous findings. ${ }^{12,27}$ The same behavior is observed in the $(21,0)$ zigzag nanotube. Nonuniform deformations, as in wrinkled nanotubes, bring water molecules closer to each other, favoring the formation of hydrogen bonds as one can see in Fig. 4(b). This effect is even more prominent in kneaded nanotubes, in which

(a)

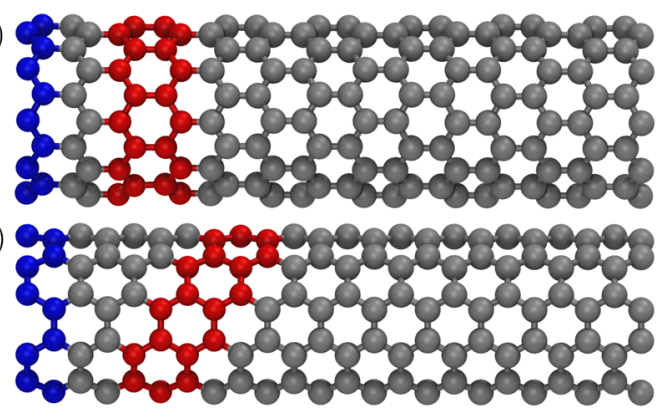

FIG. 3. Snapshot of carbon nanotube: (a) zigzag and (b) armchair. The structure highlighted in red refers to the zigzag carbon rings and armchair spiral-like structure. 
(a)

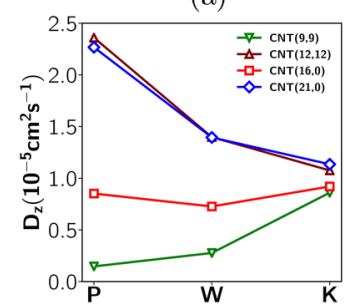

(b)

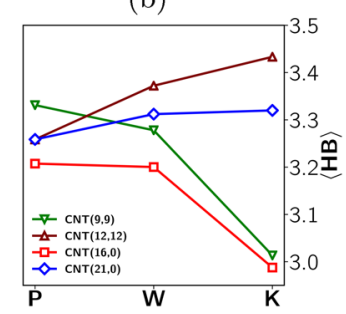

FIG. 4. (a) Axial diffusion constant and (b) average number of hydrogen bonds per water molecule for the $(9,9),(12,12),(16,0)$, and $(21,0)$ nanotubes. For the perfect, $\mathrm{P}(n, m)$; wrinkled, $\mathrm{W}(n, m)$; and kneaded, $\mathrm{K}(n, m)$ cases.

deformation and decrease in distance between molecules are more uniform. Changes in water diffusion due to deformations in $(12,12)$ and $(21,0)$ tubes are very similar, and chirality seems to play a minor role in this case.

For the armchair $(9,9)$ nanotube, a different scenario is found. Water mobility increases with deformation, which breaks hydrogen bonding networks, leading to increased confined water diffusion, as can be seen in Fig. 4. For the $(16,0)$ zigzag nanotube, the water diffusion and the number of hydrogen bonds are not affected by the change from the perfect to wrinkled nanotube. When the nanotube is kneaded, however, the number of bonds is reduced and a clear decoupling between mobility and hydrogen bond network disruption is observed. The behavior of the diffusion coefficient and
HB of water confined at the $(9,9)$ and $(16,0)$ perfect and wrinkled nanotubes is quite distinct, which indicates that for this small diameter surface effects are indeed relevant. However, for the kneaded nanotube, the water diffusion and the number of hydrogen bonds for both $(9,9)$ and $(16,0)$ nanotubes are almost the same, indicating that the strong deformation shows a stronger impact on the mobility than chirality.

In order to understand the structural origin of the difference in mobility and HB between wrinkled $(9,9)$ and $(16,0)$ nanotubes, we analyzed $x y, x z$, and $y z$ colormaps. Figure 5 illustrates these three colormaps for the water inside the wrinkled $(9,9)$ nanotube. For $25 \mathrm{~nm}$ length, the nanotube presents perfect segments with no deformation followed by deformed structures ranging from different values of $e$, as indicated by Eq. (1). Water in a perfect segment is evenly distributed, as observed at $0<z<25 \mathrm{~nm}$ in Fig. 7, what is consistent with spiral-like behavior with molecules bonded along the spiral. As the nanotube becomes deformed, these bondings are broken and the molecules in these segments form bonds in the $x y$ and $x z$ directions, forming structures quite similar to the perfect $(16,0)$ nanotube. For very strong deformation, the $x y$ bonding is destroyed and the molecules form bonded lines in the $x z$ plane.

For the $(16,0)$ wrinkled nanotube shown in Fig. 6, the perfect and not strongly deformed segments show a very similar structure, what explains why the random deformation has a very little impact on the mobility and number of hydrogen bonds. The particles show the same structure of bonds and circular mobility observed in the perfect zigzag nanotube.

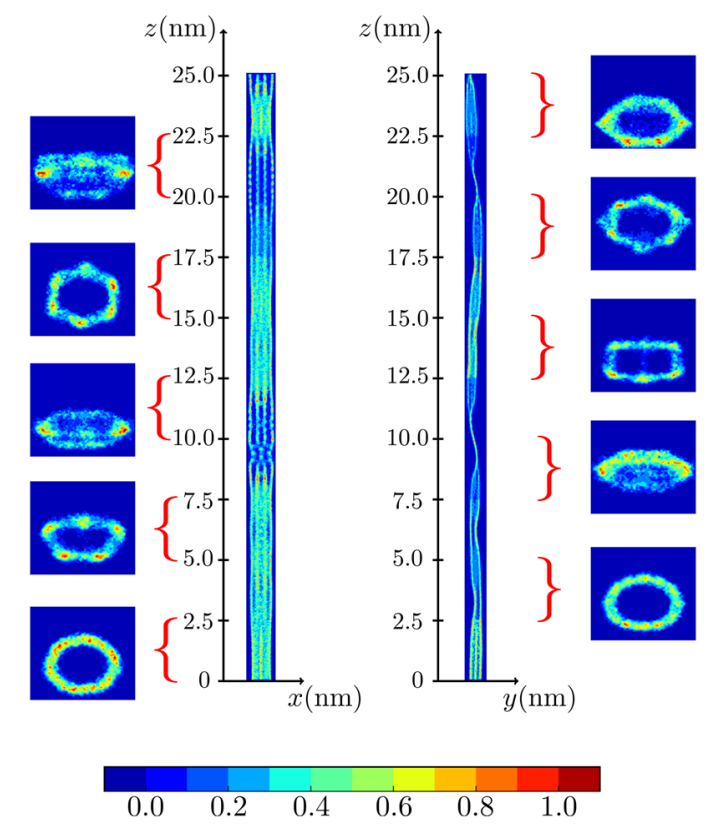

FIG. 5. Colormaps of $x z, y z$, and $x y$ directions for the $(9,9)$ wrinkled nanotube. The $x y$ colormaps are dependent on the nanotube region, so each $x y$ colormap is related to one nanotube region with $25 \mathrm{~nm}$ in the $z$ direction. Dark blue regions have low probability to find water molecules, while red regions have high probability to find water molecules.

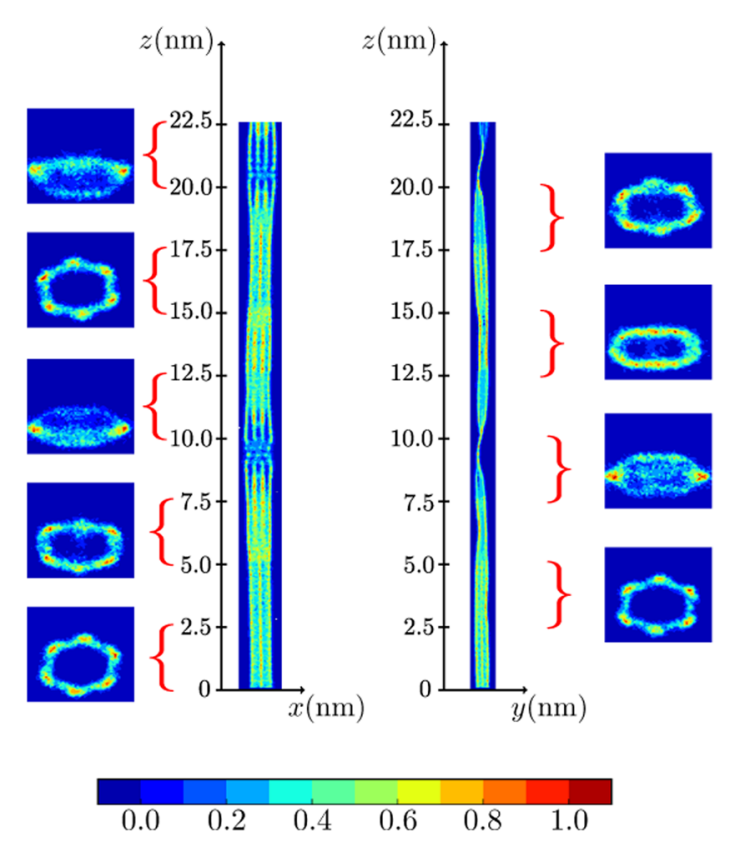

FIG. 6. Colormaps of $x z, y z$, and $x y$ directions for the $(16,0)$ wrinkled nanotube. The $x y$ colormaps are dependent on the nanotube region, so each $x y$ colormap is related to one nanotube region with $22.5 \mathrm{~nm}$ in the $z$ direction. Dark blue regions have low probability to find water molecules, while red regions have high probability to find water molecules. 




FIG. 7. Colormaps of $x z, y z$, and $x y$ directions for the $(9,9)$ kneaded nanotube, $e=0.8$. The $x y$ colormaps are dependent on the nanotube region, so each $x y$ colormap is related to one nanotube region with $25 \mathrm{~nm}$ in the $z$ direction. Dark blue regions have low probability to find water molecules, while red regions have high probability to find water molecules.

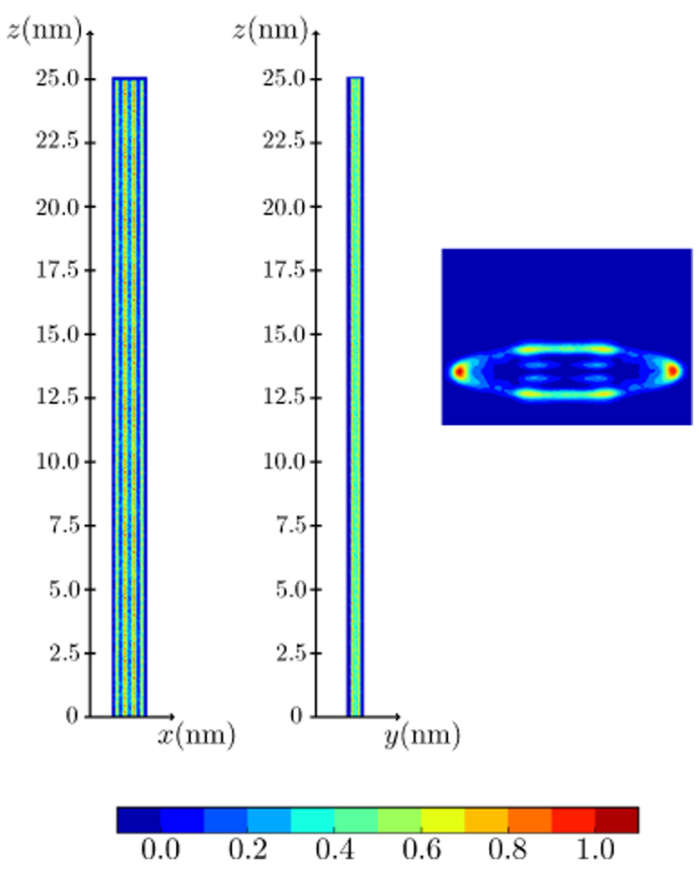

FIG. 8. Colormaps of $x z, y z$, and $x y$ directions for the $(16,0)$ kneaded nanotube, $e=0.8$. The $x y$ colormaps are dependent on the nanotube region, so each $x y$ colormap is related to one nanotube region with $25 \mathrm{~nm}$ in the $z$ direction. Dark blue regions have low probability to find water molecules, while red regions have high probability to find water molecules.
For the kneaded armchair and zigzag deformed nanotubes, the water diffusion and hydrogen bond structure are almost the same. This reflects the structure of the $(9,9)$ and $(16,0)$ tubes illustrated in Figs. 7 and 8, respectively. The deformation changes the way water molecules connect and move inside the armchair nanotube from spiral-like to circular as the water melts inside the tube.

\section{CONCLUSIONS}

In this work, we analyzed the diffusion coefficient of water under confinement in carbon nanotubes. Different tube sizes, topology, and deformation were considered.

For the perfect nanotube, the mobility of water in both armchair and zigzag topologies is very similar, with the exception of the $(9,9)$ and the $(16,0)$ cases. The water has a glassy state at $(9,9)$ while still moving at $(16,0)$, although both have the same diameter, which is attributed to the spiral and ring-shaped structure of the tubes, respectively.

As the nanotube is deformed, compression leads to a decrease in diffusion of water and an increase in the number of hydrogen bonds between water molecules for both armchair and zigzag cases for larger tubes, $(12,12)$ and $(21,0)$, respectively. For smaller tubes, $(9,9)$ and $(16,0)$, the behavior is more complex since they form a single layer of water and the water-wall interactions become relevant. For the kneaded armchair $(9,9)$ nanotube, the deformation melts water, creating a structure similar to that observed in the $(16,0)$ nanotube.

Water molecules confined within deformed, wrinkled, and dented nanotubes show a decrease in mobility and an increase in the hydrogen bonding network. For the mobility water confined, the nanotube topology is relevant only for a specific diameter (about $1.22 \mathrm{~nm}$ for the armchair and $1.24 \mathrm{~nm}$ for the zigzag), for which for which the water confined at the perfect $\operatorname{armchair}(9,9)$ nanotube features a glassy state. In this case, the deformation causes the water to come out of a state of almost zero mobility and leads to behavior similar to that observed in zigzag nanotubes.

\section{ACKNOWLEDGMENTS}

This work was partially supported by the Brazilian science agencies $\mathrm{CNPq}$ (through INCT-Fcx), CAPES, FAPEMIG, Universidade Federal de Ouro Preto, Universidade Federal do Rio Grande do Sul, and Centro Nacional de Processamento de Alto Desempenho (CENAPAD).

\section{REFERENCES}

${ }^{1}$ M. A. Ratner and D. Ratner, Nanotechnology: A Gentle Introduction to the Next Big Idea (Prentice Hall Professional, 2003), Vol. 1, p. 133.

${ }^{2}$ T. O. Fonseca, M. M. Szortyka, P. Ternes, C. Gavazzoni, A. B. de Oliveira, and M. C. Barbosa, Sci. China, Phys., Mech. Astron. 62, 107009 (2019).

${ }^{3}$ G. Hummer, J. C. Rasaiah, and J. P. Noworyta, Nature 414, 188 (2001).

${ }^{4}$ M. Majumder, N. Chopra, R. Andrews, and B. J. Hinds, Nature 438, 44 (2005).

${ }^{5}$ M. Whitby and N. Quirke, Nat. Nanotechnol. 2, 87 (2007).

${ }^{6}$ R. El-Ganainy, K. G. Makris, M. Khajavikhan, Z. H. Musslimani, S. Rotter, and D. N. Christodoulides, Nat. Phys. 14, 11 (2018).

${ }^{7}$ J. K. Holt, A. Noy, T. Huser, D. Eaglesham, and O. Bakajin, Nano Lett. 4, 2245 (2004).

${ }^{8}$ S. Li, W. Lew, J. Bland, L. Lopez-Diaz, M. Natali, C. Vaz, and Y. Chen, Nature 415, 600 (2002) 
${ }^{9}$ A. Alexiadis and S. Kassinos, Chem. Rev. 108, 5014 (2008).

${ }^{10}$ K. R. Harris and L. A. Woolf, J. Chem. Soc., Faraday Trans. 1 76, 377 (1980).

${ }^{11}$ Y.-C. Liu, J.-W. Shen, K. E. Gubbins, J. D. Moore, T. Wu, and Q. Wang, Phys. Rev. B 77, 125438 (2008).

${ }^{12}$ A. Barati Farimani and N. Aluru, J. Phys. Chem. B 115, 12145 (2011).

${ }^{13}$ Y.-g. Zheng, H.-f. Ye, Z.-q. Zhang, and H.-w. Zhang, Phys. Chem. Chem. Phys. 14, 964 (2012)

${ }^{14}$ M. H. Köhler, J. R. Bordin, C. F. de Matos, and M. C. Barbosa, Chem. Eng. Sci. 203, 54 (2019).

${ }^{15}$ M. H. Köhler and C. Gavazzoni, J. Phys. Chem. C 123, 13968 (2019).

${ }^{16}$ J. Wang, Y. Zhu, J. Zhou, and X.-H. Lu, Phys. Chem. Chem. Phys. 6, 829 (2004).

${ }^{17}$ A. I. Kolesnikov, J.-M. Zanotti, C.-K. Loong, P. Thiyagarajan, A. P. Moravsky, R. O. Loutfy, and C. J. Burnham, Phys. Rev. Lett. 93, 035503 (2004).

${ }^{18}$ N. Naguib, H. Ye, Y. Gogotsi, A. G. Yazicioglu, C. M. Megaridis, and M. Yoshimura, Nano Lett. 4, 2237 (2004).

${ }^{19}$ E. Mamontov, C. Burnham, S.-H. Chen, A. Moravsky, C.-K. Loong, N. De Souza, and A. Kolesnikov, J. Chem. Phys. 124, 194703 (2006).

${ }^{20}$ C. Y. Won, S. Joseph, and N. Aluru, J. Chem. Phys. 125, 114701 (2006).

${ }^{21}$ Q. Wang, Int. J. Solids Struct. 41, 5451 (2004).
${ }^{22}$ R. Ansari, M. Mirnezhad, and S. Sahmani, Meccanica 48, 1355 (2013).

${ }^{23}$ Y. Liu, Q. Wang, L. Zhang, and T. Wu, Langmuir 21, 12025 (2005).

${ }^{24}$ B. Xu, Y. Li, T. Park, and X. Chen, J. Chem. Phys. 135, 144703 (2011).

${ }^{25}$ G. Arora, N. J. Wagner, and S. I. Sandler, Langmuir 20, 6268 (2004).

${ }^{26}$ A. B. de Oliveira, H. Chacham, J. S. Soares, T. M. Manhabosco, H. F. de Resende, and R. J. Batista, Carbon 96, 616 (2016).

${ }^{27}$ B. H. S. Mendonça, D. N. de Freitas, M. H. Köhler, R. J. C. Batista, M. C. Barbosa, and A. B. de Oliveira, Physica A 517, 491 (2019).

${ }^{28}$ S. Iijima, Nature 354, 56 (1991).

${ }^{29}$ S. Iijima and T. Ichihashi, Nature 363, 603 (1993).

${ }^{30}$ J. L. F. Abascal and C. Vega, J. Chem. Phys. 123, 234505 (2005).

${ }^{31}$ J. R. Bordin, A. B. de Oliveira, A. Diehl, and M. C. Barbosa, J. Chem. Phys. 137, 084504 (2012).

${ }^{32}$ S. Plimpton, J. Comput. Phys. 117, 1 (1995).

${ }^{33}$ D. Ostler, S. K. Kannam, P. J. Daivis, F. Frascoli, and B. Todd, J. Phys. Chem. C 121, 28158 (2017).

${ }^{34}$ I. Hanasaki and A. Nakatani, J. Chem. Phys. 124, 144708 (2006).

${ }^{35}$ E. Kotsalis, J. H. Walther, and P. Koumoutsakos, Int. J. Multiphase Flow 30, 995 (2004).

${ }^{36}$ S. Joseph and N. Aluru, Nano Lett. 8, 452 (2008). 